Importancia de las aguadas para el pecarí de labios blancos

(Tayassu pecari) en la Selva Maya, Guatemala

\title{
Importance of waterholes for white-lipped peccary (Tayassu pecari) in the Selva Maya, Guatemala
}

\author{
José Fernando Moreira-Ramírez ${ }^{1,4^{*}}$, Rafael Reyna-Hurtado ${ }^{1}$, Mircea Hidalgo-Mihart ${ }^{2}$, Eduardo Naranjo ${ }^{1}$, Milton C. \\ Ribeiro ${ }^{3}$, Rony García-Anleu ${ }^{4}$, Melvin Mérida ${ }^{4}$ y Gabriela Ponce-Santizo ${ }^{4}$
}

\begin{abstract}
' El Colegio de la Frontera Sur, Unidad Campeche. Avenida Rancho Polígono 2A, Ciudad Industrial, Lerma 24500. Ciudad de Campeche, Campeche, México. E-mail: jfmoreira@ecosur.edu.mx (JFMR), rreyna@ecosur.mx (RRH), enaranjo@ecosur.mx (EN) Tel. 9811051708 Fax: 9811273720

${ }^{2}$ División Académica de Ciencias Biológicas, Universidad Juárez Autónoma de Tabasco, km 0.5, Carretera Villahermosa-Cárdenas, 86039. Villahermosa, Tabasco, México. E-mail: mirceahidalgo1@hotmail.com (MHM)

${ }^{3}$ Departamento de Ecologia, Universidade Estadual Paulista, Rio Claro, São Paulo, 13506-900, Brasil. E-mail: mcr@rc.unesp.br (MR) ${ }^{4}$ Wildlife Conservation Society, programa para Guatemala. Avenida 15 de marzo casa 3, Flores. Petén, Guatemala. E-mail: rgarcia@ wcs.org (RGA), mmerida@wcs.org (MM), gponce@wcs.org (GPS)

${ }^{*}$ Corresponding author
\end{abstract}

Water is considered an essential nutrient for wildlife and, when not in the proper proportion, can be a limiting factor to populations. Differences in water dependency among ungulate species can arise through a variety of physiological, morphological and behavioral mechanisms employed in maintaining the balance of temperature and water. The white-lipped peccary forms large and cohesive groups of 10 to over 300 individuals inhabiting dense tropical forests. Our objectives were to describe the use of waterholes by whitelipped peccaries groups in a humid site of the Selva Maya and evaluate the effect of humidity may have on the visit frequency, group size and activity patterns. From June to August 2014 and February to April 2015 seven waterholes were monitored using digital camera traps. The visit frequency was estimated by dividing the number of events between 1,000 sampling effort traps night. The minimum group size and age structure were estimated for each separate event. Activity patterns were estimated at one-hour intervals for the dry and rainy seasons. Forty-seven and 185 separate events for the rainy and dry season respectively were obtained. The sampling effort in each period was 630 traps night. For the rainy and dry season we obtained a visit frequency of 74.6 and 293.7 respectively. For the rainy and dry season an average group size of $17( \pm 9.5)$ and $25.5( \pm 12.6)$ were estimated respectively. The groups are mainly composed of adults. The presence of newborns was mainly in August and April. Activity patterns were mainly recorded between 10:00 h and 16:00 $\mathrm{h}$. The visit frequency estimated is higher compared to other protected areas in the Selva Maya. The minimum group size estimated is similar to those reported in dry areas within the Selva Maya. The presence of newborns was reported during all months of the study, existing peaks during August, March and April. The white-lipped peccary visited the waterholes mainly during the day between 10:00 $\mathrm{h}$ and 16:00 h. Waterholes in the Laguna del Tigre National Park can be called "sanctuaries" for white-lipped peccaries because are extremely important in the ecology of this social ungulate.

El agua es considerada un nutriente esencial para la vida silvestre siendo un factor de bienestar y cuando no está en la proporción adecuada, puede ser un factor limitante para las poblaciones silvestres. Las diferencias en la dependencia del agua entre las especies de ungulados pueden surgir a través de la variedad de mecanismos fisiológicos, morfológicos y conductuales empleadas en el mantenimiento del equilibrio de la temperatura y el agua. El pecarí de labios blancos es un ungulado social que forma grupos grandes y cohesivos entre 10 a más de 300 individuos habitando en selvas tropicales densas. Nuestros objetivos fueron describir el uso de las aguadas por los grupos de pecarí de labios blancos en un sitio húmedo de la Selva Maya y evaluar el efecto que la humedad puede ejercer sobre la frecuencia de visita, tamaño de grupo y patrones de actividad. De junio a agosto del 2014 y de febrero a abril del 2015 fueron monitoreadas siete aguadas con trampas cámara digitales. La frecuencia de visita fue estimada dividiendo el número de eventos entre el esfuerzo de muestreo por 1,000 trampas noche. El tamaño mínimo de grupo se estimó por cada evento independiente registrando adultos, subadultos y crías. Los patrones de actividad se estimaron en intervalos de una horas, para la época seca y lluviosa. Se obtuvieron 47 y 185 eventos independientes para la época lluviosa y seca, respectivamente. El esfuerzo de muestreo en cada época fue de 630 trampas noche. Para la época lluviosa y seca obtuvimos una frecuencia de visita de 74.6 y 293.7 respectivamente. Para la época lluviosa y seca se estimó un tamaño promedio de grupo de 17 ( \pm 9.5$)$ y 25.5 ( \pm 12.6 ) respectivamente. Los grupos están compuestos principalmente por adultos. La presencia de crías se registró principalmente en agosto, marzo y abril. Los patrones de actividad se registraron principalmente entre las 10:00 h y 16:00 h.: La frecuencia de visita estimada es más alta comparada con otras áreas protegidas de la Selva Maya. El tamaño mínimo de grupo estimado es similar a los reportados en áreas secas dentro de la Selva Maya. La presencia de crías fue reportada durante todos los meses del estudio, existiendo picos en los 
meses de agosto, marzo y abril. El pecarí de labios blancos visitó las aguadas principalmente durante el día entre las 10:00 y 16:00 h. Las aguadas dentro del Parque Nacional Laguna del Tigre pueden ser denominadas "santuarios" para el pecarí de labios blancos ya que son sumamente importantes en la ecología de este ungulado social.

Key words: Births season; Camera traps; conservation; Guatemala; Selva Maya; ungulate.

๑) 2016 Asociación Mexicana de Mastozoología, www.mastozoologiamexicana.org

\section{Introducción}

El agua es considerada un nutriente esencial para la vida silvestre siendo un factor de bienestar (Leopold 1933; Krausman y Etchberger 1995; Cain et al. 2006) y cuando no está en la proporción adecuada, puede ser un factor limitante para las poblaciones silvestres (Krausman et al. 2006). Existen tres formas generales en que el agua puede estar disponible para la vida silvestre: 1) agua pre-formada que está disponible en los alimentos, 2) agua metabólica que se crea como un sub-producto de procesos bioquímicos (e. g. metabolismo de la grasa o descomposición de los carbohidratos) y 3) el agua libre que se encuentra disponible para beber (Robbins 2001). Las diferencias en la dependencia del agua entre las especies de ungulados pueden surgir a través de la variedad de mecanismos fisiológicos, morfológicos y conductuales empleadas en el mantenimiento del equilibrio de la temperatura y el agua (Cain et al. 2006). El pecarí de labios blancos, es un ungulado altamente frugívoro de amplia distribución que forma grupos grandes y cohesivos desde 10 a más de 300 individuos adaptado a una gran variedad de ambientes, pero principalmente en selvas tropicales densas (Sowls 1997; Fragoso 1998; Beck 2006; MoreiraRamírez et al. 2015). En general, la ingesta diaria de agua en pecaríes es principalmente derivada de sus alimentos, pero se complementa con agua libre obtenida en el ambiente (Sowls 1997). Esta necesidad ha hecho que el pecarí de labios blancos modifique notablemente su comportamiento, especialmente en sitios donde el agua no se encuentra homogéneamente distribuida en el tiempo y el espacio.

Los mamíferos neotropicales varían en su tendencia a seguir o cruzar senderos y esto tiene implicaciones en la probabilidad de detección de las especies utilizando métodos como trampas cámara (Harmsen et al. 2009). En Amapá, Brasil, utilizando trampas cámara en senderos en un área de $25 \mathrm{~km}^{2}$ con un esfuerzo de 1,800 trampas noche no fue detectada ninguna fotocaptura de pecarí de labios blancos (Michalski et al. 2015). Este patrón también ha sido observado en el Parque Nacional Laguna del Tigre, donde solamente se obtuvieron siete fotocapturas utilizando trampas cámara en senderos (Moreira-Ramírez et al. 2009). Esto puede deberse a que esta especie presenta baja detección en caminos y porque tienen ámbitos de hogar grandes por lo que se requiere de mayores esfuerzos de muestreo (Tobler et al. 2008; Michalski et al. 2015). Sin embargo, estudios previos muestran que esta especie presenta alta detección en cuerpos de agua favoreciendo el monitoreo de esta especie en sitios clave como las aguadas (Reyna-Hurtado et al. 2010a; Moreira-Ramírez y García 2011; Pérez-Cortez et al. 2012).

La distribución histórica del pecarí de labios blancos en Guatemala hasta el 2005 se ha reducido en un $84 \%$ (Altrichter et al. 2012). La Reserva de la Biosfera Maya es la única área protegida en donde aún permanece una población viable (Altrichter et al. 2012). En áreas donde esta especie tiene una mayor presión de caza, son comunes los grupos pequeños de 10 individuos (Emmons y Feer 1990; March 1993). Debido a los impactos que tiene sobre sus poblaciones, a la pérdida de hábitat, cacería y transmisión de enfermedades la especie se encuentra registrada en el apéndice II del Convenio sobre Comercio Internacional de Especies Amenazadas de Fauna y Flora Silvestre (CITES 2006) y como "Vulnerable" por la Lista Roja de la Unión Mundial para la Naturaleza en todo su ámbito de distribución (Fragoso 1997; Altrichter et al. 2012; Keuroghlian et al. 2013). 
En la Selva Maya los suelos son de origen calcáreo, lo que ocasiona que el agua de las lluvias se filtre rápidamente al subsuelo por lo que no existen cuerpos de agua permanentes (ReynaHurtado et al. 2010a). Sin embargo, hay zonas donde las características del suelo permiten la acumulación de la precipitación, lo que forma cuerpos de agua conocidos localmente como aguadas (Reyna-Hurtado et al. 2010a). Las aguadas son sumamente importantes para la vida silvestre, ya que proveen de agua superficial a diferentes especies, entre ellas al pecarí de labios blancos (Moreira-Ramírez y García 2011) y al tapir centroamericano (Tapirus bairdii; Pérez-Cortez et al. 2012). En la región de Calakmul, se registró que las aguadas representan el elemento del paisaje más importante para los movimientos del pecarí de labios blancos (Reyna-Hurtado et al. 2009). Debido a la necesitad que presenta el pecarí de labios blancos por obtener agua y a la heterogeneidad espacial de la disponibilidad de este recurso, se ha encontrado que los grupos de esta especie recorrieron distancias de hasta $17 \mathrm{~km}$ en dos días para acceder a cuerpos de agua (Reyna-Hurtado et al. 2009). Además, las variables ambientales que más influyeron en la abundancia del tapir en Calakmul fueron el porcentaje de agua presente en las aguadas y la disponibilidad de frutos alrededor de estos cuerpos de agua (Pérez-Cortez et al. 2012). Investigaciones previas documentaron el tamaño de grupo, composición y patrones de actividad del pecarí de labios blancos en áreas secas de la Selva Maya con precipitación promedio menor a los 1,300 mm (Moreira-Ramírez y García 2011; Moreira-Ramírez et al. 2015; Naranjo et al. 2015). Sin embargo estas variables no han sido medidas en lugares húmedos de la Selva Maya.

Para cuantificar el uso de las aguadas por el pecarí de labios blancos, utilizamos trampas cámara digitales para registrar la frecuencia de visita, tamaño de grupo, temporada de crías y patrones de actividad. Nuestros objetivos fueron: describir el uso de las aguadas por los grupos de pecarí de labios blancos en un sitio húmedo de la Selva Maya y evaluar el efecto que este factor puede ejercer sobre la frecuencia de visita, tamaño de grupo y patrones de actividad durante la época seca y lluviosa.

\section{Materiales y Métodos}

Área de estudio. El Parque Nacional Laguna del Tigre (PNLT) se localiza al oeste de la Reserva de la Biosfera Maya (RBM; Figura 1). El PNLT se encuentra ubicado entre las coordenadas $17^{\circ} 10^{\prime} 30^{\prime \prime}$ $\mathrm{N},-90^{\circ} 02^{\prime} 44^{\prime \prime} \mathrm{W}$ y $17^{\circ} 19^{\prime} 49^{\prime \prime} \mathrm{N},-90^{\circ} 25^{\prime} 22^{\prime \prime} \mathrm{W}$. El área fue declarada como Parque Nacional en 1990 con el establecimiento de la Reserva de la Biosfera Maya, cubriendo 337,899 hectáreas. La temperatura promedio en la época seca y época lluviosa es de $35^{\circ} \mathrm{C}$ y $25^{\circ} \mathrm{C}$ respectivamente. La precipitación promedio anual se encuentra entre 1,400 a $1,796 \mathrm{~mm}$ y la altitud varía entre los 40 a 200 m (CONAP 2007). Existen dos épocas climáticas marcadas. La época seca abarca de febrero a mayo y la lluviosa, de junio a enero (CONAP 2007). Los tipos de vegetación predominantes en la parte este del parque son selva mediana subperennifolia (árboles entre 15-25 m de altura), selva mediana y baja semidecidua (árboles entre 8-25 m de altura) y selva baja subperennifolia inundable (árboles entre 5-15 m de altura, CONAP 2007). Las especies de árboles más importantes que se ubican en el área de estudio son ramón (Brosimum alicastrum), en asociación con zapotillo (Pouteria reticulata), silión ( $P$. amygdalina), canisté ( $P$. campechiana) y chicozapote (Manilkara zapota). El PNLT está divido en tres subcuencas principales: río Candelaria, río San Pedro y río Escondido (CONAP 2007). Se estima que existen al menos 680 cuerpos de agua, lo que equivale al $70 \%$ de todos los cuerpos de agua de la Reserva de la Biosfera Maya (CONAP 2007).

Las amenazas principales para el PNLT son la deforestación, los incendios forestales causados principalmente por los asentamientos humanos no planificados y las actividades petroleras sin mitigación. El aumento en la red vial ligada a la actividad petrolera ha sido un factor fundamental para el ingreso de nuevas familias originando nuevos asentamientos. La mayor parte de los 
asentamientos están distribuidos en la zona central, aledañas a la carretera principal construida por la petrolera (CONAP 2007; CONAP-WCS 2015).

Monitoreo de aguadas. Durante los meses de junio a agosto del 2014 (época lluviosa) y de febrero a abril del 2015 (época seca) fueron monitoreadas siete aguadas (Figura 1). Los criterios para seleccionar las aguadas fueron presencia de agua, presencia de caminos hechos por el pecarí de labios blancos, y presencia de lodo. La distancia promedio entre las aguadas fue de $2.2 \mathrm{~km}(0.7-3.7 \mathrm{~km})$. Alrededor de las aguadas se encuentran principalmente árboles de pucté (Bucida buceras), zapote bobo (Pachira aquatica), escobo (Chrysophila argentea) y guano (Sabal morrisiana). Las siete aguadas siempre presentaron presencia de lodo y agua durante los meses que duró el muestreo, abarcando la época lluviosa y seca. En cada aguada fue colocada una trampa cámara digital. Utilizamos trampas cámara digitales marca Reconyx (RapidFire HC800 , www.reconyx.com) y Bushnell (www.bushnell.com/hunting/trail-cameras) las cuales toman imágenes de alta calidad, no poseen flash o luz blanca que podría interferir en el comportamiento de algunas especies y además poseen el retraso de disparo más corto que cualquier otro equipo similar ( $0.2 \mathrm{~s}$ para las Reconyx y $0.8 \mathrm{~s}$ para las Bushnell). Las trampas cámara fueron ubicadas en la orilla de la aguada, en lugares con presencia de caminos y/o bañaderos para aumentar la probabilidad de fotografiar a los pecaríes de labios blancos que ingresaban al cuerpo de agua, para estimar de mejor manera el tamaño mínimo de los grupos, composición, frecuencia de visita y patrones de actividad. Las trampas cámara fueron colocadas a una altura entre 40 a 50 $\mathrm{cm}$ amarradas a un árbol. Se programaron para tomar series de tres fotografías por detección de evento, estando activas durante las $24 \mathrm{~h}$ del día. Cada trampa cámara fue referenciada con un GPS marca Garmin ${ }^{\circledR}$ modelo 62 s y revisada una vez al mes para constatar que el funcionamiento de la cámara y baterías fueran los adecuados y a su vez para almacenar la información capturada y vaciar la memoria de la misma.

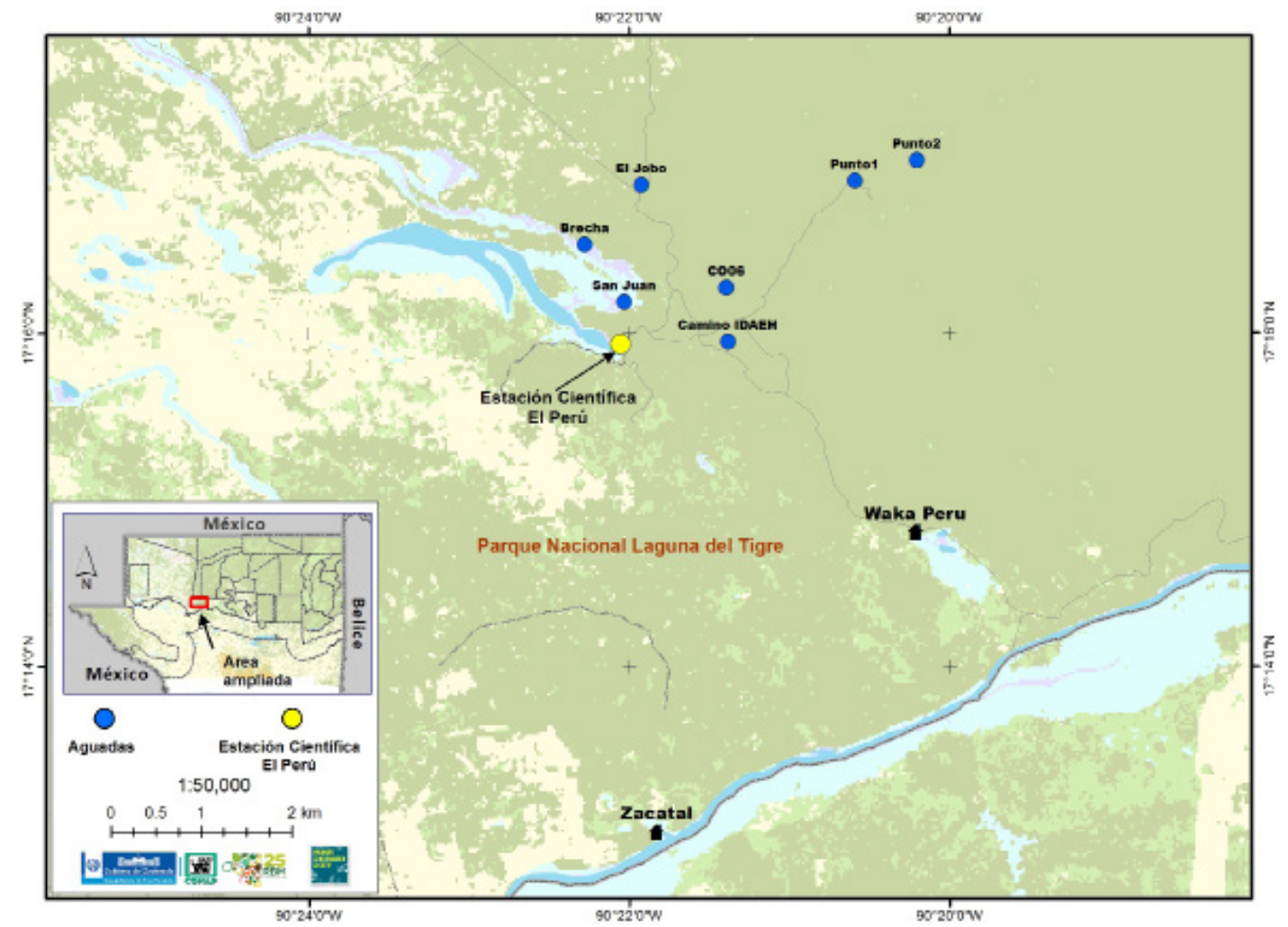

Figura 1. Mapa de ubicación geográfica del Parque Nacional Laguna del Tigre y ubicación de las trampas cámaras en siete aguadas (color azul), Guatemala. 
Índice de frecuencia de visita. Para obtener el índice de frecuencia de visita (IFV) del pecarí de labios blancos en el área se utilizó la siguiente fórmula (Maffei et al. 2002; Lira-Torres et al. 2014): IFV $=(C / E M) \times 1,000$ trampas noche. Donde: $C$ es el número de capturas o eventos fotográficos independientes; EM es el esfuerzo de muestreo (medido como número de trampas cámara multiplicado por las noches de monitoreo, por época y por mes de muestreo) y 1,000 noches trampa (unidad estándar). Utilizamos este índice para comparar las frecuencias de visita de los grupos durante cada mes y época del año. Para la época lluviosa y seca se detectaron grupos de pecarí de labios blancos en 33 y 55 días respectivamente. Para considerar un registro como fotocaptura independiente, se seleccionaron los grupos de imágenes de pecarí de labios blancos que presentaban como mínimo 30 minutos entre cada registro en la misma aguada (O’Brein et al. 2003; Bowkett et al. 2007; Michalski 2010). Por medio de las fotocapturas obtenidas de dos individuos marcados con collares en un grupo de pecaríes se ha observado que este grupo visita las aguadas en promedio una vez por día. Esta información ha servido para separar los registros independientes de las visitas de los grupos de pecaríes a las aguadas cada 30 minutos.

Tamaño de grupo, composición y temporada de crías. Por medio de las secuencias de fotografías obtenidas con las trampas cámara se estimó el tamaño mínimo de cada grupo que visitó las aguadas. Al momento de observar cada secuencia de fotografías se registró en la base de datos la fecha, número de fotografía, hora de entrada y de salida del grupo de pecaríes a la aguada, número de crías, subadultos, adultos y el total de individuos para la época seca y lluviosa (Figura 2). Al registrar la hora de entrada y de salida de cada grupo a la aguada estimamos el tiempo promedio de visita para la época seca y lluviosa. Para identificar crías, subadultos y adultos se utilizaron los siguientes criterios: las crías son de tamaño pequeño comparado con los subadultos y adultos, son de color café claro y se desplazan junto a sus madres. Los subadultos presentan tamaños intermedios y son de color café claro negruzco. Los adultos son de color negro y presentan el mentón de color blanco (Sowls 1984, 1997; Moreira-Ramírez et al. 2015).

Patrones de actividad. Los eventos fotográficos de pecarí de labios blancos fueron agrupados en una hoja de cálculo y se estimó la frecuencia de visitas a lo largo de 24 horas divididas en lapsos de una hora para la época seca y lluviosa (Cuellar y Noss 2003). El tiempo promedio de duración de la visita a las aguadas por los grupos de pecaríes de labios blancos fue estimado por medio de la hora registrada durante el ingreso y salida de cada evento fotográfico independiente.

Análisis de datos. Se utilizó una prueba de $U$ de Mann-Whitney para comparar la frecuencia de visita y el tamaño de grupo entre la época lluviosa y seca. Se utilizó la frecuencia de visita por mes para evaluar la variación temporal mensual de visita a las aguadas por el pecarí de labios blancos. Para esta evaluación se usó la prueba no paramétrica de $X^{2}$ de Pearson. Para todas las pruebas estadísticas se usó un nivel de significancia de $P<0.05$. Las variables que se midieron fueron: frecuencia de visita por época y por mes, número de individuos (adultos, subadultos y crías) por grupo y número total tanto para la época seca y lluviosa. Los descriptores de la composición de los grupos se expresaron como: porcentaje de adultos, subadultos y crías. Las variables de la temporada de crías se expresaron como porcentaje de fotografías independientes por mes. Los descriptores de organización social fueron: tamaño de los grupos, la media del tamaño y composición ( \pm desviación estándar) de grupos. Los análisis estadísticos fueron realizados utilizando el programa R 2.13.1 (R Development Core Team 2011). 


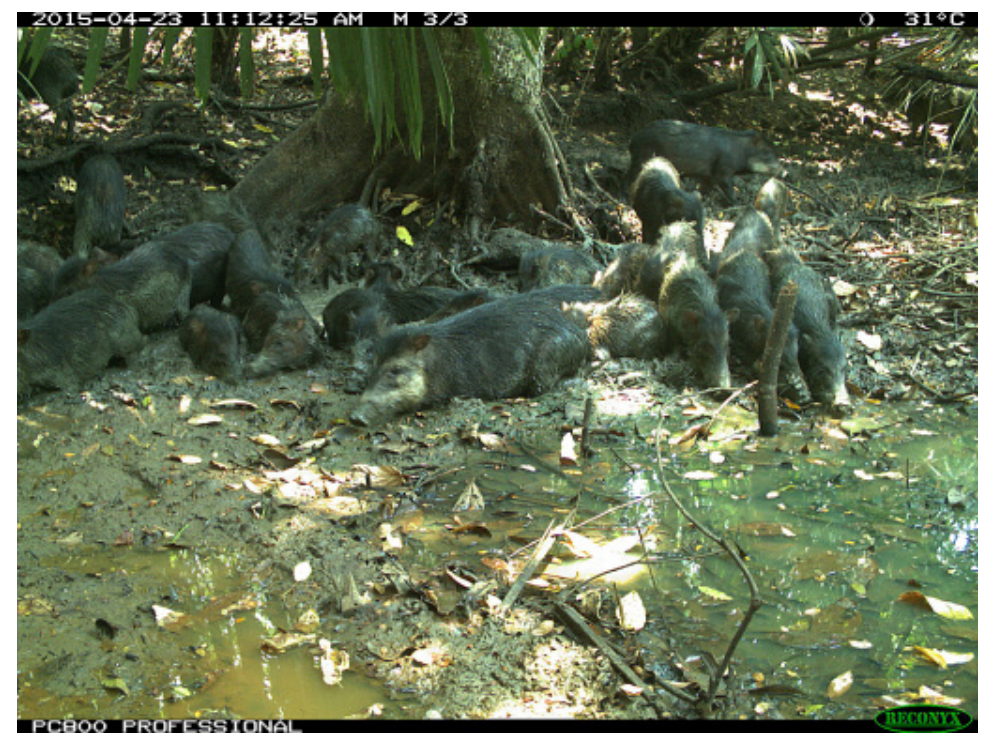

Figura 2. Individuos de pecarí de labios blancos (Tayassu pecari) ingresando a una aguada. Cada fotografía presenta la fecha, hora, número de la secuencia. Parque Nacional Laguna del Tigre, Guatemala.

\section{Resultados}

El esfuerzo de muestreo en cada época fue de 630 trampas noche (210 trampas noche por mes) obteniéndose 47 y 185 fotografías independientes para la época lluviosa y seca respectivamente.

Frecuencia de visita. Para la época lluviosa y seca obtuvimos un índice de frecuencia de visita de 74.6 y 293.7 , respectivamente. La frecuencia de visita estimada para la época seca fue mayor comparada con la época lluviosa $(U=410.5, P<0.01)$. La visita a las aguadas por el pecarí de labios blancos, medida por la frecuencia de visita mensual, varió entre cada mes ( $x 2=33$, d. f. $=5, P<0.001$; Figura 3 ). La frecuencia de visita más alta estimada en la época lluviosa y en la época seca se dio en los meses de agosto y abril respectivamente (Figura 3).

Tamaño de grupo, composición y temporada de crías. Para la época lluviosa y seca se estimaron tamaños promedio de grupo de $17( \pm 9.5, n=47)$ y $25.5( \pm 12.6, n=185)$ respectivamente. El tamaño de los grupos fue mayor en la época seca en comparación con la época lluviosa $(U=2566, P=0.001)$.

Los grupos de pecarí de labios blancos están compuestos principalmente por adultos. Para la época lluviosa se encontró que los adultos componen el $69 \%$ ( $n=$ 536), los subadultos componen el $10 \%(n=81)$ y las crías componen el $21 \%(n=$ 160). Para la época seca se encontró que los adultos componen el $83 \%(n=3,881)$, los subadultos componen el $7 \%(n=327)$ y las crías componen el $10 \%(n=477)$. Para la época lluviosa y seca se observaron 24 y 7 grupos respectivamente compuestos solamente por adultos. Tanto para la época lluviosa como seca se observó mayor proporción de crías comparado con los subadultos.

La presencia de crías durante la época lluviosa se da principalmente en agosto representando el $61 \%$ (eventos con crías $=22$ ), seguido por julio con el $22 \%$ (eventos con crías $=8$ ) y junio con el $17 \%$ (eventos con crías $=6$ ). En la época seca la presencia de crías se registró principalmente en abril representando el $63 \%$ (eventos con crías $=93$ ), seguido por marzo con el $31 \%$ (eventos con crías $=45$ ) y febrero con el $6 \%$ (eventos con crías $=9$; Figura 4). 


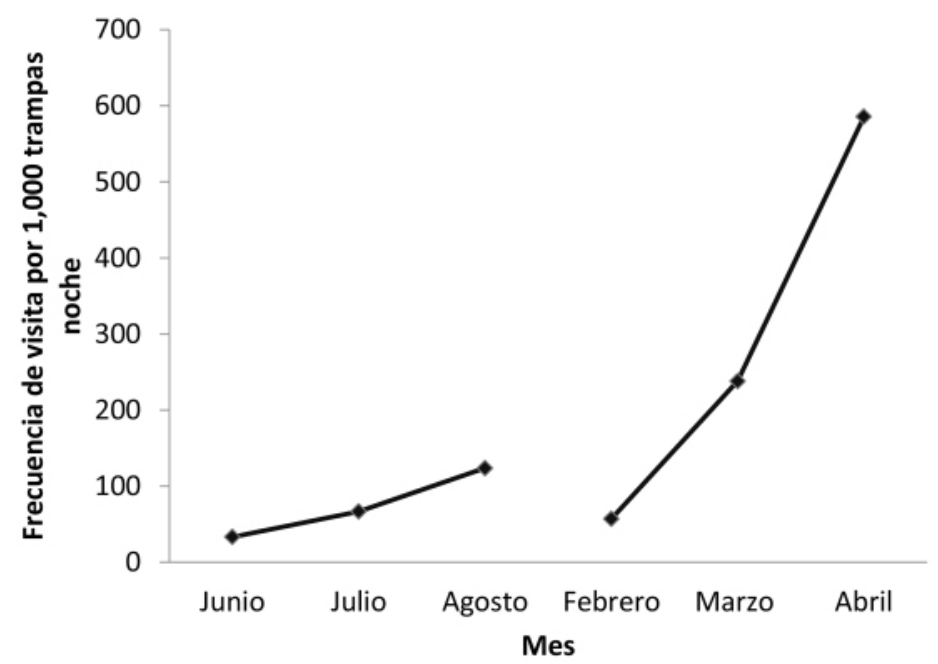

Figura 3. Frecuencia de visita por mes del pecarí de labios blancos en aguadas estimada por trampas cámara.

Patrones de actividad. Los patrones de actividad del pecarí de labios blancos en las aguadas fueron principalmente diurnos. Para la época lluviosa el $42.5 \%$ (28) de las fotografías independientes se registraron entre las 10:00 h y 14:00 h. Para la época seca el $54.6 \%$ (101) de las fotografías independientes se registraron entre las 10:00 h y 16:00 h. Además para esta época se registró una leve actividad en las aguadas durante la noche, entre las 20:00 h y 22:00 h (7; Figura 5).

Los grupos de pecarí de labios blancos pasaron más tiempo en las aguadas durante la época seca en comparación con la lluviosa. El tiempo promedio de visita a las aguadas fue de 19 min y 54 segundos para la época seca y de 14 min y 56 segundos para la época lluviosa.

\section{Discusión}

Las aguadas situadas en el interior del bosque tropical al este del Parque Nacional Laguna del Tigre son un recurso importante para el pecarí de labios blancos como se muestra por el número de fotografías independientes obtenidas en siete cuerpos de agua diferentes estudiados en los meses de junio a agosto de 2014 y febrero a abril del 2015. Estudios previos utilizando trampas cámara en senderos dentro del bosque muestran bajas frecuencias de captura del pecarí de labios blancos (Moreira-Ramírez et al. 2007), incluso en algunos sitios bien conservados del noreste de la Amazonia brasileña, con esfuerzos de muestreo de 1,800 trampas noche, no se registraron grupos de esta especie (Michalski et al. 2015).

La frecuencia de visita estimada para la época seca fue mayor comparada con la época lluviosa. Este patrón también ha sido observado para el Parque Nacional Mirador-Río Azul (PNMRA; Moreira-Ramírez 2009). Esto puede deberse a que durante la época seca el pecarí de labios blancos visita las aguadas con mayor frecuencia. La frecuencia de visita más alta estimada en la época lluviosa y en la época seca se dio en los meses de agosto y abril respectivamente. Una mayor frecuencia de visita en estos dos meses puede deberse a la alta temperatura promedio registrada. El promedio de temperatura máxima para el mes de agosto fue de $36.5^{\circ} \mathrm{C}$ y para el mes de abril de 39.5 ${ }^{\circ} \mathrm{C}$ (Estación Meteorológica Mactún, San Andrés, Petén). En la Reserva de la Biosfera 


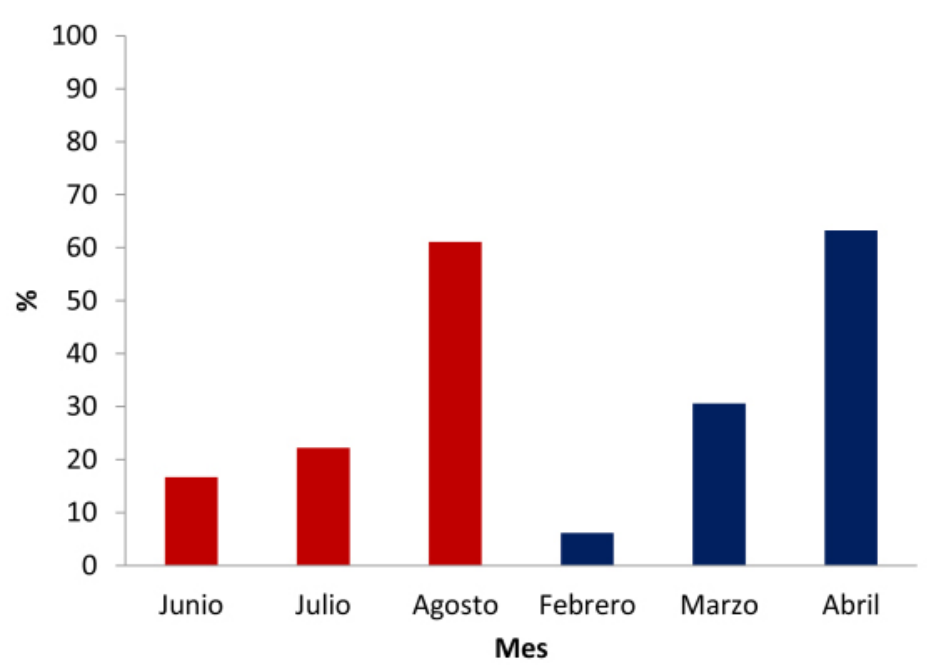

Figura 4. Presencia de crías de pecarí de labios blancos en tres meses de la época lluviosa (año 2014,color rojo) y tres meses de la época seca (año 2015, color azul) en el Parque Nacional Laguna del Tigre, Guatemala.

Calakmul (RBC), los pecaríes de labios blancos aumentaron sustancialmente sus ámbitos de hogar cuando la época de lluvias llegó, lo que sugiere que la disponibilidad de agua juega un papel importante en la determinación de los movimientos de esta especie (Reyna-Hurtado et al. 2009). Las frecuencias de visita estimadas en nuestro estudio (74.6 - 293.7) fueron mayores comparadas con las registradas en aguadas en la RBC (14.2; Naranjo et al. 2015) y en senderos y/o caminos en los Chimalapas (37.6; Lira-Torres y Briones-Salas 2012), Selva Lacandona (4.2) y Balam-Kin (13.3; Naranjo et al. 2015), similares a las estimadas en el sureste de Perú (66 - 82.5; Tobler et al. 2009) y menores a las estimadas en el Parque Nacional Madidi, Bolivia (488.9; Gomez et al. 2005). Las frecuencias de visita estimadas en aguadas del PNLT se encuentran dentro de los intervalos reportados para otras áreas protegidas de Mesoamerica. Debido a esto es importante mantener las brigadas de protección conformadas por personal del Consejo Nacional de Áreas Protegidas y de organizaciones no gubernamentales para continuar con los patrullajes dentro del parque para evitar la cacería, incursión de asentamientos humanos ilegales e incendios forestales, sobre todo en años muy calurosos por el efecto de El Niño (INSIVUMEH 2015).

El tamaño promedio de los grupos presentó una tendencia a ser mayor en la época seca comparada con la lluviosa. Esta diferencia puede deberse a que los grupos visitan con mayor frecuencia las aguadas durante la época seca, limitando los ámbitos hogareños de los grupos a estos cuerpos de agua (Reyna-Hurtado et al. 2009). Durante la época lluviosa los grupos tienden a ser más móviles y a desplazarse largas distancias (Fragoso 1998; Reyna-Hurtado et al. 2009). Otra posibilidad es que esta diferencia se deba a intercambios entre individuos lo cual ha sido reportado en estudios realizados en el Parque Nacional Corcovado, Costa Rica (Carrillo et al. 2002) y en el bosque Atlántico en Brasil (Keuroghlian et al. 2004). Estos autores sugieren que las manadas forman una "súper-manada" la cual a la vez está integrada por varias "sub-manadas". Esta característica en la biología de las manadas de jabalí dentro del PNLT solamente puede ser evaluada por medio de estudios con radiotelemetría, lo cual podrá dar información acerca de la existencia o no de intercambios de individuos. 


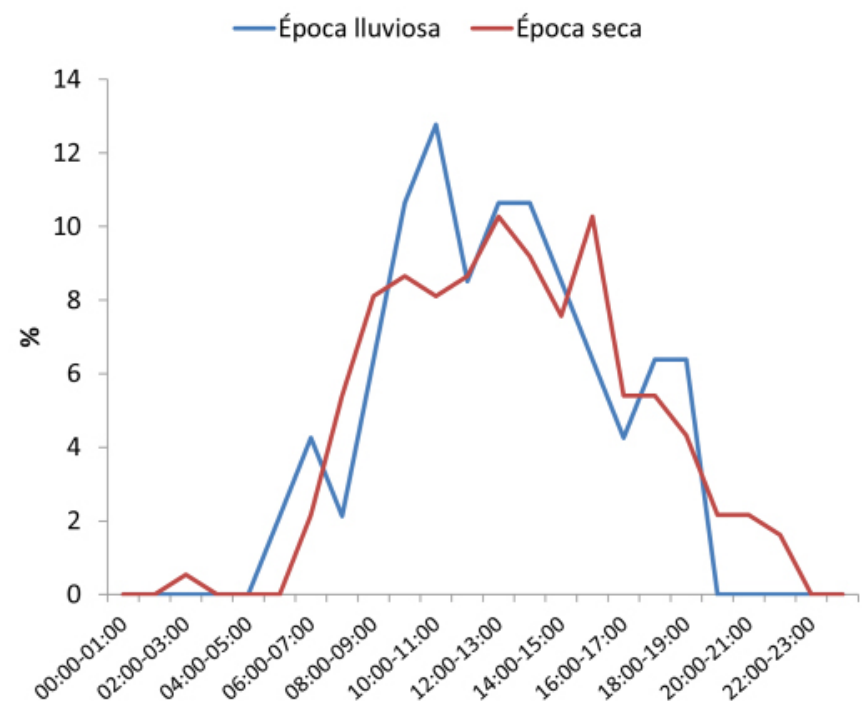

Figura 5. Patrones de actividad de pecarí de labios blancos en las aguadas registrados por medio de trampas cámara durante la época lluviosa $(n=47)$ y época seca $(n=185)$.

Los tamaños promedio de los grupos del PNLT fueron similares a los estimados con trampas cámara en el Biotopo Protegido Dos Lagunas (BPDL; 27; Moreira-Ramírez y García 2011) y a los estimados por observaciones directas en la RBC (25; ReynaHurtado et al. 2010b), Parque Nacional Mirador-Rio Azul (31; Moreira-Ramírez et al. 2015), el Chaco en Argentina (23, Altrichter 2005). Los tamaños de grupo fueron menores a estimaciones realizadas en la Isla de Maracá, Brasil (114; Fragoso 1998) y en el Parque Nacional Manú, Perú (106; Kiltie y Terborgh 1983). El tamaño de grupo del pecarí de labios blancos en el PNLT se encuentra dentro de los intervalos estimados para Mesoamérica, en áreas donde no existe cacería (Reyna-Hurtado et al. 2015). Esto demuestra que la población de pecarí de labios blancos en el este del PNLT no se encuentra afectada por esta actividad humana. Durante las revisiones de las trampas cámara en las aguadas no encontramos indicios de presencia de cazadores en esta parte del Parque. Sin embargo será importante continuar con los patrullajes conjuntos liderados por el Consejo Nacional de Áreas Protegidas para evitar incursiones de cazadores y de nuevos asentamientos humanos. Además, se debe de continuar con el trabajo social y de concientización en la aldea de Paso Caballos para que los habitantes controlen las quemas utilizadas para preparar la tierra para cultivar, sobre todo durante la época seca.

La composición de los grupos de pecarí de labios blancos reportada para el PNLT fue similar a la registrada en otras partes de la Selva Maya (Reyna-Hurtado et al. 2010b; Moreira-Ramírez et al. 2015). Durante la época lluviosa y seca se observó la presencia de las tres clases de edades. El $70 \%$ de los grupos estuvo formado por adultos. Durante la época lluviosa se observó una mayor proporción de subadultos y de crías comparada con la época seca. Los grupos de la época seca tuvieron una mayor proporción de adultos comparada con la época lluviosa.

La presencia de crías en el PNLT fue registrada durante todos los meses que comprendió el estudio, de junio a agosto del 2014 y de febrero a abril del 2015. Durante la época lluviosa y la época seca el mayor porcentaje de eventos con cría se dio en los meses de agosto $(61 \%, n=22)$ y abril $(63 \%, n=93)$ respectivamente. Si tomamos en cuenta que el período de gestación es en promedio de 158 días (entre 156 y 162 días; 
Sowls 1984). El pecarí de labios blancos se aparea durante los meses de noviembre y marzo en el PNLT. Esta información coincide en parte con lo reportado para la RBC donde la temporada de crías se da principalmente en la época seca, entre los meses de enero y mayo (Reyna-Hurtado et al. 2010b). Esta variación en la temporada de crías y de apareamiento puede estar influenciada por la disponibilidad de alimento y presencia de agua (Sowls 1997). En la Guyana Francesa (Henry 1994) y Texas (Sowls 1984) la precipitación es un importante factor en la regulación reproductiva del pecarí de collar (Pecari tajacu). Dan a luz principalmente durante la época de lluvias debido a las diferencias ambientales drásticas en la disponibilidad de recursos entre los períodos húmedos y secos. La información registrada en el PNLT muestra que el pecarí de labios blancos utiliza las dos épocas del año para reproducirse, por tal motivo la cacería de esta especie no debe de ser permitida durante estos periodos. El normativo de la ley de caza en Guatemala debe de ser discutido para que las cuotas de cacería autorizadas para el pecarí de labios bancos no afecten la sobrevivencia de la especie. Esto permitirá que la especie logre recuperarse en áreas que han sido gravemente afectadas por la cacería en la zona de usos múltiples de la RBM, en los límites de parque nacionales y concesiones comunitarias. Instituciones de gobierno como el Consejo Nacional de Áreas Protegidas, investigadores, organizaciones no gubernamentales y comunitarias, deben de tomar esta iniciativa para desarrollar planes de manejo para esta especie (Moreira-Ramírez et al. 2015).

Los patrones de actividad del pecarí de labios blancos en aguadas dentro del PNLT muestran la misma tendencia entre la época seca y lluviosa. El pecarí de labios blancos visitó las aguadas principalmente durante el día, tanto en la época lluviosa como en la época seca. Los picos de actividad se dieron principalmente entre las 10:00 h y 16:00 h. Este mismo patrón ha sido reportado en el bosque del Chaco y la Chiquitanía, en Bolivia utilizando trampas cámara en salitrales (Maffei et al. 2002; Cuellar y Noss 2003) y en aguadas en el PNMRA (Moreira-Ramírez et al. 2015). Además se registró actividad durante la noche en la época seca, entre las 20:00 h y 22:00 h. Durante la época seca la temperatura ambiental diaria puede sobre pasar los $38^{\circ} \mathrm{C}$, incluso en la noche. Esto hace que los grupos de pecaríes visiten las aguadas con mayor frecuencia para darse baños de lodo para protegerse del exceso de calor y de ectoparásitos, tomar agua y tomar siestas en las inmediaciones de las aguadas. En contraste con lo reportado para el PNMRA, los pecaríes de labios blancos visitaron las aguadas durante más tiempo. Para la época seca permanecieron en promedio 19 minutos (máximo 1 h y 30 minutos) y durante la época lluviosa 15 minutos (máximo $1 \mathrm{~h})$.

Implicaciones para la conservación. En Guatemala, el único lugar en donde aún permanece una población viable de pecarí de labios blancos es dentro de la RBM. Se estima que esta reserva contiene una población residente de pecarí de labios blancos suficientemente grande para ser potencialmente auto-sostenible en los próximos 100 años, con hábitat adecuado y una diversa base alimentaria (Altrichter et al. 2012). Sin embargo el PNLT está considerado como una de las zonas núcleo de la RBM críticas para la conservación debido a las fuertes amenazas que enfrenta. Las amenazas principales para este parque son la deforestación, los incendios forestales causados principalmente por los asentamientos humanos no planificados, la búsqueda de áreas para cultivo, ganadería extensiva y las actividades petroleras sin mitigación (CONAP 2007; CONAP-WCS 2015). Debido a esto el CONAP y organizaciones no gubernamentales han implementado estrategias de protección, sobre todo en el 
este del PNLT, manteniendo presencia interinstitucional en diferentes campamentos reduciendo las amenazas en los últimos ocho años. Estas actividades han permitido que especies altamente amenazadas como el pecarí de labios blancos sobrevivan dentro de esta área protegida. Nuestro estudio demuestra que las frecuencias de visita y tamaños de grupo del pecarí de labios blancos son relativamente altos comparados con otras áreas protegidas de la Selva Maya. Los tamaños de los grupos estimados en nuestro estudio son similares a los observados en áreas protegidas mejor conservadas y más secas de la RBM que no presentan incendios forestales anuales y cambios drásticos en la cobertura forestal, como el PNMRA y el BPDL. Además, las aguadas dentro del PNLT podrían ser consideradas "santuarios" para el pecarí de labios blancos siendo sumamente importantes en la ecología de este ungulado social. Se considera necesario continuar con el monitoreo en estos sitios clave para comprender de mejor manera los factores que influyen en el uso de determinadas aguadas y los beneficios obtenidos del suelo y del agua.

\section{Agradecimientos}

A Wildlife Conservation Society, Programa para Guatemala, en especial a R. Balas por el apoyo brindado. Al Consejo Nacional de Ciencia y Tecnología de México por la beca número 308055 brindada a JFMR. A American Society of Mammalogists, Wildlife Conservation Society, programa para Guatemala, El Colegio de la Frontera Sur, Unidad Campeche, Universidad de McGill y a Idea Wild por el soporte financiero y donación de equipo. Al Consejo Nacional de Áreas Protegidas y al Instituto de Antropología e Historia por el apoyo, permisos y las facilidades brindadas. A K. Tut, P. Pérez, C. Umaña, Y. Polanco, A. Xol, R. Chatá y Y. López por la ayuda en campo y a los voluntarios que colaboraron en la instalación y revisión de las trampas cámara. A N. Solis por su ayuda en la elaboración del mapa.

\section{Literatura citada}

Altrichter, M., A. Taber, H. Beck, R. Reyna-Hurtado, L. Lizarraga, A. Keuroghlian, y E. Sanderson. 2012. Range-wide declines of a key Neotropical ecosystem architect, the Near Threatened white-lipped peccary Tayassu pecari. Oryx 46:87-98.

Beck, H. 2006. A review of peccary-palm interactions and their ecological ramifications across the Neotropics. Journal of Mammalogy 87:519-530.

Bowkett, A. E., F. Rovero, y A. R. Marshall. 2007. The use of camera trap data to model habitat use by antelope species in the Udzungwa Mountain forest, Tanazania. African Journal of Ecology 46:479-487.

Cain III, J. W., P. R. Krausman, S. S. Rosenstock, y J. C. Turner. 2006. Mechanisms of thermoregulation and water balance in desert ungulates. Wildlife Society Bulletin 34:570-581.

Carrillo, E., J. C. Saenz, y T. K. Fuller. 2002. Movements and activities of white-lipped peccaries in Corcovado National Park, Costa Rica. Biological Conservation 108:317-324.

Consejo Nacional de Áreas Protegidas (CONAP). 2007. Plan Maestro Parque Nacional Laguna del Tigre-Biotopo Laguna del Tigre Río Escondido PNLT-BLTRE. Ciudad de Guatemala, Guatemala.

Consejo Nacional de Áreas Protegidas y Wildlife Conservation Society (CONAP-WCS). 2015. Monitoreo de la gobernabilidad en la Reserva de la Biosfera Maya. Actualización a 2014 de la versión de septiembre de 2013. San Benito, Guatemala. 
Convention on International Trade in Endangered Species of Wild Fauna and Flora (CiTES). 2006. Consultada el 14 de enero de 2015. Disponible en: http://www.cites.org

Cuellar, E., y A. Noss. 2003. Mamíferos del Chaco y de la Chiquitana de Santa Cruz, Bolivia. Editorial Fan. Santa Cruz de la Sierra, Bolivia.

De la CRUz, J. 1982. Clasificación de zonas de vida de Guatemala a nivel de reconocimiento. Instituto Nacional Forestal, Ministerio de Agricultura, Ganadería y Alimentación. Ciudad de Guatemala, Guatemala.

Desbiez, A.L.J., S.A. Santos, A. Keuroghlian, y R. Bodmer. 2009. Niche partitioning among white-lipped peccaries (Tayassu pecari), collared peccaries (Pecari tajacu), and feral pigs (Sus scrofa). Journal of Mammalogy 90:119-128.

Emmons, L. H., y F. Feer. 1990. Neotropical Rainforest Mammals: A Field Guide. The University of Chicago Press. Chicago, EE. UU.

Fragoso, J. M. V. 1997 . Desapariciones locales del baquiro labiado (Tayassu pecari) en la Amazonía: migración, sobre-cosecha o epidemia? Pp. 309-312 en Manejo de fauna silvestre en la Amazonía (Fang, T., R. Bodmer, R. Aquino, y M. Valqui, eds.). United Nations Development Program-Global Environmental Facility, Universidad de Florida, Instituto de Ecología. La Paz, Bolivia.

Fragoso, J. M. V. 1998. Home range and movement patterns of white-lipped peccary (Tayassu pecari) herds in the northern Brazilian Amazon. Biotropica 30:458-469.

Gómez, H., R. Wallace, G. Ayala y R. Tejada. 2005. Dry season activity periods of some Amazonian mammals. Studies on Neotropical Fauna and Environment 40:91:95.

Harmsen, B., R. J. Foster, S. Silver, L. Ostro, y C.P. Doncaster. 2009. Differential Use of Trails by Forest Mammals and the Implications for Camera-Trap Studies: A Case Study from Belize. Biotropica 42:126-133.

Henry, O. 1994. Saisons de reproduction chez tres Rongeurs et un Ariodactyle en Guyane FrancËaise, en fonction des facteurs du milieu et de l'alimentation. Mammalia 58:183-200.

instituto Nacional de Sismología, Vulcanología, Meteorología e Hidrología (INSiVUMeh). 2015. Evolución del fenómeno de El Niño y la perspectiva climática. Boletín climático número 10-2015. Ciudad de Guatemala, Guatemala.

Keuroghlian, A., D. P. Eaton, y W. S. Longland. 2004. Area use by white-lipped and collared peccaries (Tayassu pecari and Tayassu tajacu) in a tropical forest fragment. Biological Conservation 120:411-425.

Keuroghlian, A., A. Desbiez, R. Reyna-Hurtado, M. Altrichter, H. Beck, A. Taber, y J. M. Fragoso. 2013. Tayassu pecari. IUCN Red List of Threatened Species. Consultada el 10 de marzo de 2015. Disponible en: http//www.iucnredlist.org

KitTIE, R. A., Y J. TeRBORGH. 1983. Observations on the behavior of rain forest peccaries in Perú: why do white-lipped peccaries form herds? Zeitschrift fur Tierpsychologie 62:241-255.

Krausman, P., y R. C. Etchberger. 1995. Response of desert ungulates to a water project in Arizona. Journal of Wildlife Management 59:292-300.

Krausman, P., S. S. Rosenstock, y J. W. Cain III. 2006. Developed Waters for Wildlife: Science, Perception, Values, and Controversy. Wildlife Society Bulletin 34:563-569.

Leopold, A. S. 1933. Game management. Charles Scribner's Sons. New York, EE. UU.

LiRA-Torres, I., y M. Briones-Salas. 2012. Abundancia relativa y patrones de actividad de los mamíferos de los Chimalapas, Oaxaca, México. Acta Zoológica Mexicana (n. s.) 28:566-585.

Lira-Torres, I., M. Briones-Salas, y G. Sánchez-Rojas. 2014. Abundancia relativa, estructura poblacional, preferencia de hábitat y patrones de actividad del tapir Centroamericano Tapirus bairdii (Perissodactyla: Tapiridae), en la Selva de Los Chimalapas, Oaxaca, México. Revista de Biología Tropical 62:1407-1419. 
Maffel, L., E. CuellaR, y J. Noss. 2002. Uso de trampas cámara para la evaluación demamíferos en el ecotono Chaco-Chiquitanía. Revista Boliviana de Ecología y Conservación Ambiental 11:55-65.

MarCH, I. 1993. The white lipped peccary (Tayassu pecari). Pp. 13-22 en Pigs, peccaries and hippos: status survey and conservation plan, IUCN (Oliver, W., ed.). UICN Gland, Suiza.

Michalskı, F. 2010. The bush dog Speothos venaticus and short-eared dog Atelocynus microtis in a fragmented landscape in southern Amazonia. Oryx 44:300-303.

Michalski, J.L, D. Norris, T. Oliveira, y F. Michalski. 2015. Ecological Relationships of MesoScale Distribution in 25 Neotropical Vertebrate Species. PLoS ONE 10(5):e0126114.

Moreira-Ramírez, J.F., R. McNab, D. Thornton, R. García, G. Ponce-Santizo, y J. Radachowsky. 2007. Abundancia de Jaguares en La Gloria-El Lechugal, Zona de Usos Múltiples, Reserva de la Biosfera Maya, Petén, Guatemala. Informe interno. Wildlife Conservation Society. Flores, Guatemala.

Moreira-Ramírez, J. F., R. McNab, R. García, G. Ponce, M. Mérida, V. Méndez, M. Córdova, G. Ruano, y K. Tut. 2009. Abundancia y densidad de Jaguares en El Parque Nacional Laguna del Tigre- Corredor Biológico Central, Reserva de la Biosfera Maya. Informe interno. Wildlife Conservation Society. Flores, Petén, Guatemala.

Moreira-Ramírez, J.F.. 2009. Patrones diarios de actividad, composición, tamaño y abundancia relativa de manadas de jabalí Tayassu pecari (Link, 1795), en el Parque Nacional Mirador-Río Azul, Petén, Guatemala. Tesis de Licenciatura. Universidad de San Carlos de Guatemala. Ciudad de Guatemala, Guatemala.

Moreira-Ramírez, J.F., y R. García. 2011. Uso de trampas cámara digitales para estudiar al Jabalí (Tayassu pecari) en el Biotopo Protegido Dos Lagunas, Reserva de la Biosfera Maya. Suiform Soundings 10:32-37.

Moreira-Ramírez, J.F., J.E. López, R. García-Anleu, F. Córdova, y T. Dubón. 2015.Tamaño, composición y patrones diarios de actividad de grupos de pecarí de labios blancos (Tayassu pecari) en el Parque Nacional Mirador-Río Azul, Guatemala. Therya 6:469-482.

Naranjo, E., S. Amador-Alcalá, F. Falconi-Briones, y R. Reyna-Hurtado. 2015. Distribución, abundancia y amenazas a las poblaciones de tapir centroamericano (Tapirus bairdii) y pecarí de labios blancos (Tayassu pecari) en México. Therya 6:227-249.

O'Brein, T., M.F. GKInNARID, y H.T. WiBisono. 2003. Crouching tigers, hidden prey: Sumatran tiger and prey populations in a tropical forest landscape. Animal Conservation 6:131-139.

Pérez-Cortez, S., P. Enríquez, D. Sima-Panti, R. Reyna-Hurtado, y E. Naranjo. 2012.Influencia de la disponibilidad de agua en la presencia y abundancia de Tapirus bairdii en la selva de Calakmul, Campeche, México. Revista Mexicana de Biodiversidad 83:753-761.

Reyna-Hurtado, R., E. Rojas-Flores, y G. Tanner. 2009. Home range and habitat preferences of white-lipped peccaries (Tayassu pecari) in Calakmul, Campeche, México. Journal of Mammalogy 90:1199-1209.

Reyna-Hurtado, R., G. O'Farril, D. Sima, M. Andrade, A. Padilla, y L. Sosa. 2010a. Las aguadas de Calakmul, reservorios de fauna Silvestre y de la riqueza natural de México. Biodiversitas 93:1-6.

Reyna-Hurtado, R., E. Naranjo, C. A. Chapman, y G. Tanner. 2010b. Hunting patterns, population density, group size, and conservation of the white-lipped peccary (Tayassu pecari) in the Calakmul region of Mexico. Oryx 44:88-96.

Reyna-Hurtado, R., H. Beck, M. Altrichter, C. A. Chapman, T. R. Bonnell, A. Keuroghlian, A. Desbiez, J. F. Moreira-Ramírez, G. O’Farril, J. Fragoso, y E. Naranjo. 2015. What ecological and anthropogenic factors affect group size in white-lipped peccaries (Tayassu pecari)? Biotropica 0:1-9. 
R Development Core Team. 2011. R: A language and environment for statistical computing. $R$ Foundation for Statistical Computing, Vienna, Austria. ISBN 3-900051-07-0, URL http:// www.R-project.org

Roвbins, C. T. 2001. Wildlife feeding and nutrition. Academic Press. San Diego, EE. UU.

SowLs, L. 1984. The peccaries. Universidad de Arizona. Press. Tucson, EE. UU.

SowLs, L. 1997. Javelinas and other Peccaries: Their, Biology, Management, and Use. The Texas A\&M Univertsity Press. College Station, EE. UU.

Tobler, M. W., S. E. Carrillo-Percastegui, R. Leite Pitman, R. Mares, y G. Powell. 2008. An evaluation of camera traps for inventorying large- and medium-sized terrestrial rainforest mammals. Animal Conservation 11:169-178.

Tobler, M. W., S. E. Carrillo-Percastegul, y G. Powell. 2009. Habitat use, activity patterns and use of mineral licks by five species of ungulate in south-eastern Peru. Journal of Tropical Ecology 25:261-270.

Submited: October 15, 2015

Reviewed: December 3, 2015

Accepted: January 10, 2016

Associated editor: Cristina Macswiney 